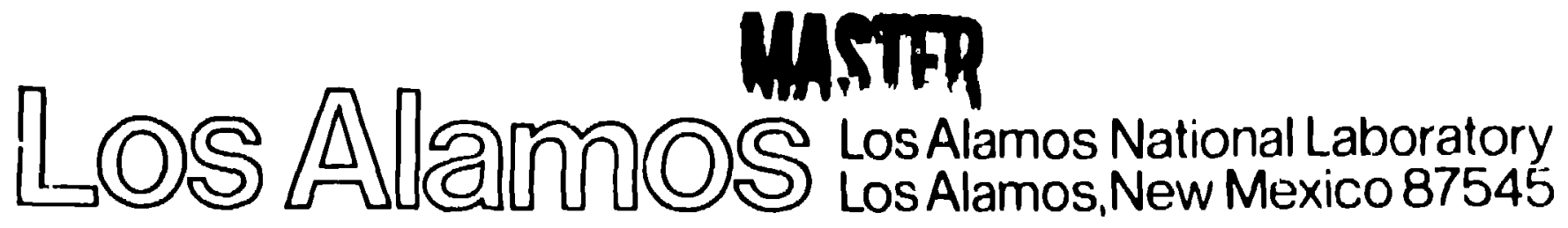




\title{
CONSTRAINTS ON STELIAR EVOLUTION FROM PULSATIONS
}

\author{
Arthur N. Cox \\ Theoretical Division, Los Alamos National Laboratory \\ University of California \\ Los Alamos, New Mexico 87545 USA
}

\begin{abstract}
Consideration of the many types of intrinsic variable stars, that is, those that pulsate, reveals that perhaps a dozen clas'es can indicate some constraints that affect the results of stellar evolution calculations, or some interpretatiors of observations. Many of these constraints are not very strong or may not even be well defined yel. In this review we discuss only the case for six classes: classical Cepheids with their measured Wesselink radii, the observed surtace effective temperatures of the known eleven double-mode Cepheids, the pulsation periods and measured surface effective temperatures of three $R$ CrB variables, the $\delta$ Scuti variable VZ Cne with a very large ratio of its two olserved periods, the nonradial oscillations of our sun, and the period ratios of the newly discnvered double-mode Rh Lyrae variables. Unfortunately, the present state of knowledge ahout the exact compositions; mass loss and its dependence on the mass, radius, luminosity, and composition; and internal mixing processes, as well as sometimes the more basic parameters such as luminosities and aurface cllective cemperatures prevent us from applying strong constraints for every case where currently the possibility exists.
\end{abstract}

Use of the pulsation existence as evidenced by observed periods is oftell of limited value becalise the actual mode of the pulantion is uncertail, for eximple, these are the questions whether sone clasidcal Cephejde are ralial fundanental or firsl overtone pulsators, or whether the |l cephei variables are radial or nonradial pulantore. This problem is Homelimes very embarrassing becouse the dispule over whether the Mira variables are radial fundamental or radial first overtolle pulate cors mealie that we cannot identlly periods that are a lactor ol three different. For most canes, however, ilie princilial mode of pulsution is well known, nlthough mily other modes present con be folentified only With lenN Ceilainty

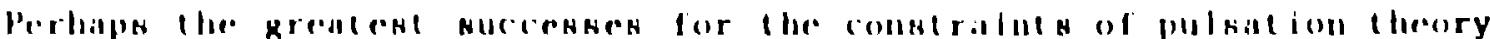

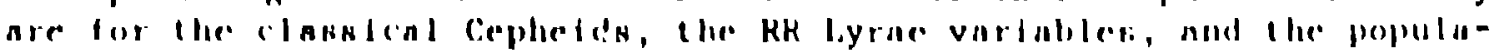
lon II Cephelds (III. Her and w Vir yarinblen). Manhen derived liy mome pilladion-haned methode agree well with evolut lon lheory, but there nre 
problems rhat still exist. These problems might eventually be bonafide constraints to be settled by changes in our current ideas of the internal composition structure. It seeins that, except for much needed accurate surface effective temperatures for Mira variables and for the extremely hot dwarf stars and more periods for double-mode $R R$ Lyrae variables in the field and in globular clusters, the next odvances in these pulsation-based constraints will come from theoretical calculations of internal mixing and element separatiou and from nonlinear pulsation calculations.

Cepheid masses have been shown by $\operatorname{Cox}$ (1979) to be consistent with evolution calculations using the new larger distance scale of Hanson (1977) and the cooler effective temperutures of Pel (1978), Flower (1977) and Bell and Parsons (1972). We ignore for this discussion the problems for the double-mode and bump Sepheids that can indicate internal helium enhancements or magnetic fields. For periods longer than $1 C$ days, there has been a remaining problem that the Wessellak masses used with the period-mean density relation produce masses too small by typically a factor of 0.6-0.7. Wi here propose as Burki (1983) recently has done, that these Wesselink radii indicate mass loss rates that can be used as a constrajnt on evolution calculations which include mass loss.

The measured Westelink radius has been discussed by Burki, who shows that the presence of a companion ster can greatly affect the derived radius. Therefore, it in beller that only truly single- Cepheids be considered. Solution of three equations, the period-mean density relation, the definition of the surface effective temperature, and a fit for the pulsation constant $Q$ as a function of mass, radius, luminosity, and effective lemperature, are solved for threc unknowns, Q, luminosity and mass when the period, effective temperature and Wesselink radius are given. The Cepheide KS Pup, I Car, U Car, and SV Vul (possibly n binary) are shown to have a mass hs small at; 0.3 the so-ralled theoretical mass. Burki shows that the Maeder (1981) evolution tracks with moderate to large mase loss (cases H and C) bracket the current masses for llese four Cepheids. Figure l gives these Hurki muser vele.is luminosity for these Cepheids together with the cane A (no mas loss), B and C relations for the initial masses of 9 nad $15 M_{H}$. It uppears that the mass loge rate nimost as high us

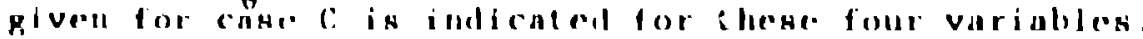

Actually both RS Pup and SV Vui have mensured luminosities and elfective lemperatures, allowing the caleulation of their pulantlon mansen. KS Pup Nhowe litele mase loss in figure 1 , and inderal the

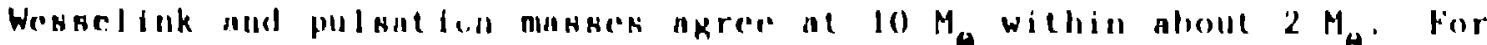
SV Vul, however, Il npperare that evolution, Pheorelienl and pulsulion

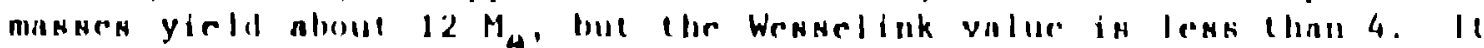
nuy lie that indered SV Vill is n binary etar with n blue compunion which wrongly reduces lis Weskelink ralius by all appreciable anoust nal

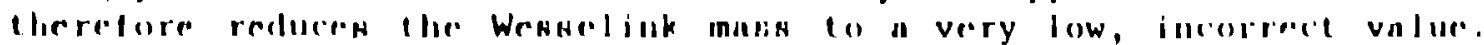
We do not have luminosity data for the two other cincian atare lo 


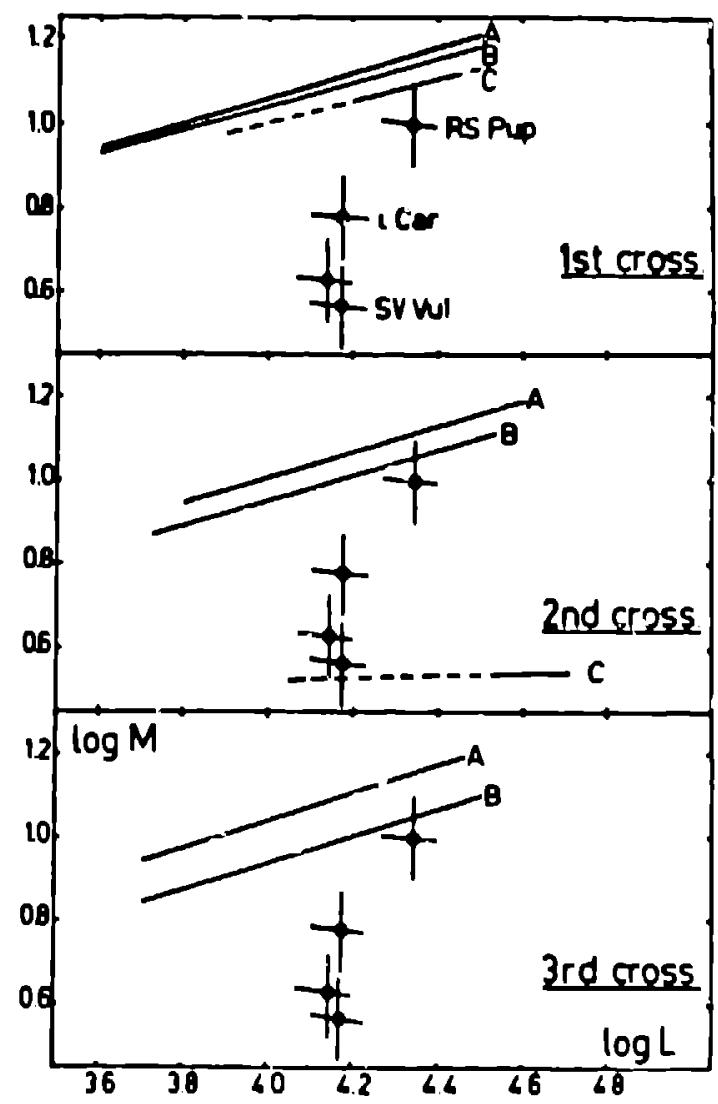

Figure 1. The luminosity-mass relation for four Cepheids with Wesselink masses and luminosities from the work of Burki. Also plotled are the Maeder theoretical relations during the first, serond, and third crossings of the Cepheid instability thrip for no mass loss (cuse A), moderate mass loss (case B) and high mass loss (case C).

dispute thes Wesselink masses, and so factors of two mass loss for long period Cepheids may be correct. This result constitules a constraint on the mass loss rales for ysllow giants of original mass hbout $12 \mathrm{M}_{\mathrm{G}}$.

Accually the observed SV Vul period decrease reported by fernic (1979) ano indirates blueward evolution that occurs slowly compared to the first and poselbly the third croseings of the instabijity strip when the original mass is aboat 15 $M_{\text {f }}$ Since evolution track: indicate very rapid bluewnrd evolulion when the mase lons rate is as high an for chae C. it is very unlikely that nuch a short-lived Cephedid would be observabje with a rupldly decreasing period. The large mass lone of case $C$ acems excluded by observitions of the modernte long period leppheid period decreanes.

Since the In rge oxygen depletion reported by luck and Lambert (1981) IIS) longer aceme to be correct, thr Iarge overshoot lng nt 9 Macheridered 
by Becker and Cox (1982) in the main sequence stages is not necessary. The result that the blue loops are at a much higher luminosity than thought before is unaffected, however, and the 15 or more day Cepheid evolution masses are reduced as much as $17 \%$ as reported by Becker and Cox and by Matraka, Wasserman and Weigert (1982).

For shorter period Cepheids, there is another problem. The two periods of the double-mode Cepheids and the bumps for the 5 - 15 day bump Cepheids suggest masses considerably less than the evolutionary, theoretical, or pulsation masses. The factor is 0.6 for the bump Cepheids, while it is about 0.3 for those double-mode Cepheids that directly display two periods. The question for the last 10 years has been what is the rause of such large theuretical $P_{1} / P_{0}$ period ratios and such low derived masses? Are they really correct or is someting else wrong? The current proposed answers are that the surface layers have an unconventional structure which produces a few percent increase of the fundamental radial mode period relative to a similar increase of the first overtone mode period. This structure can be established by an actual mass loss or an entanced helium content, a magnetic fieid, or an opacity increase in the outer $10^{-4}$ of the mass. The first and last of these suggestions now seems to be ruled out by other considerations that we will not discuss here.

Other discussion of the cause of this anomalously small period ratio, as indeed the cause of the double-mode pulsation. we leave to another talk. Here we merely look at the position of these variables on evolutionary Lracks to sec what constraints exist on evolutionary theory. Balona and Stobie (1979) made carefu' measurements of the intrinsic colors of eight of the 11 double-mode Cepheids. Conversion of these colors by a formula given by Bell and Parsons (1972), shows that all these variables have the same effective temperature of $5940 \mathrm{~K}$ within a small scatt:r of aboul. $130 \mathrm{~K}$. More recently, Barrell (1981) using the shape of the hydrogen o line, has verified both the narrow range of the temperature and its meall value for all 11 variables. Our present interest is lo see if we can learn whil this means for evolulion eracks.

The cause of blue loops that produce Cepheids is the existence of the $\mu$-gralient region left behind by a shrinking convective core ill maill fequence evolution. Too much mass loss call disrupt lhis structural feature and produce only a return lo near lie main sequence. Also tor only rather low or rather high ratios of the mixtug lenglh to pressure Heale height in the surface convection zone can these hlue loops liring the eracks into the Cepheid variable ingtability strip.

Kecently Huang nud Weigert (1983) have investignted this last anint as well as how much overshootillp in allowed from the central hydrogen burning convective core lo homogenige the bot tom part of the p-gridient shell. Pliey rind that, nt 5 M, overshooling of no more llinu hall of a presaure achle height above the convective rore is allowed or ho blue

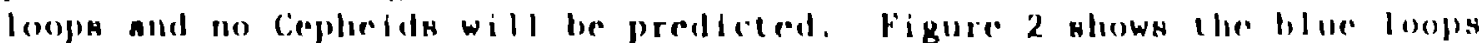
for the two cases with the core collvertion overshooting 0.5 ninl 1.0 
pressure scale heights. The effects of the outer ratio of convective mixing length to pressure scale height are also indicated tor both core overshooting cases. This limit of only about 0.5 scale height core overshooting is reasonable because there is really a very strong $\mu$-barrier against the outward motion of the heavy helium-rich core material into the lighter hyưrogen-ich layers. For normal compositions then, there are these reasonable constraints: some, but not much, core overshooting, Maeder and Mermilloid (1981), and a convective envelope ratio of mixing length to pressure scale height not much smaller than 1.5 , just as appropriate for our sun.
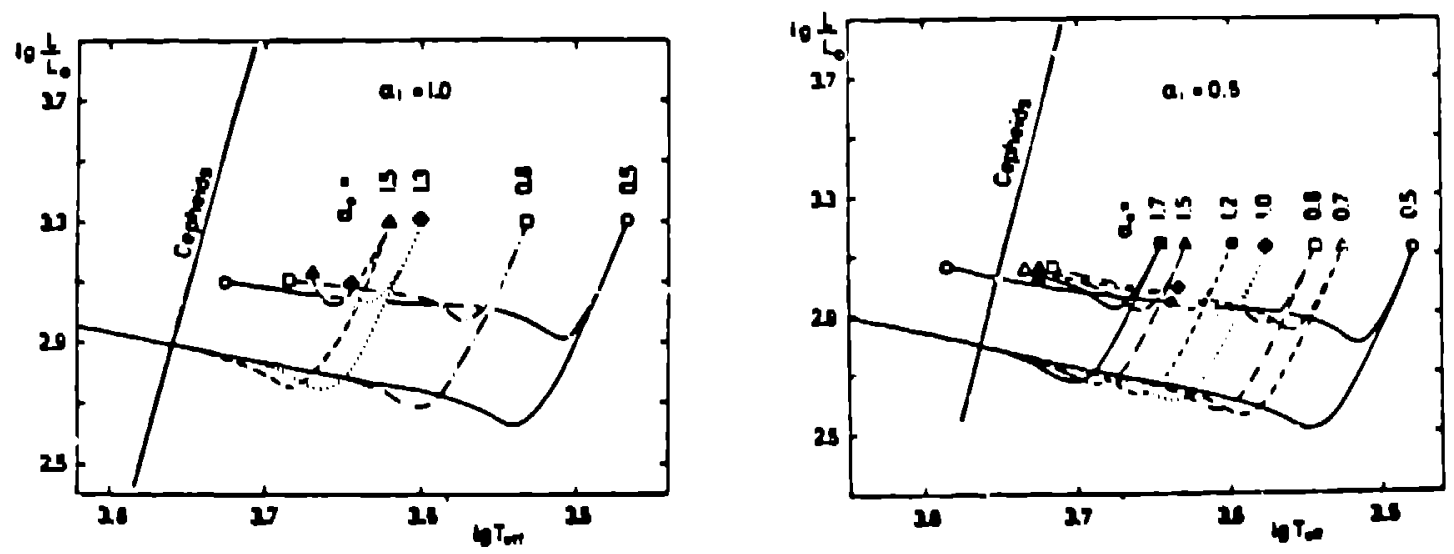

Figurc 2. Theoretical Hertzsprung-Russell diagrams are given for the core overshooting ef 1.0 and 0.5 pressure scale heights. For each case the ratio of the mixing length to pressure scale height ranges from 0.5 to 1.7 for the outer convertive shell.

Unfortundely, the length of the blue loops depends also on the composition. For low 7 , he tracks can go much bluer, but such low $Z$ values do not seem reasonable for the recently born 5 to $15 M_{0}$ Cepheids. Figure 3 shows the track of a $6.0 \mathrm{M}$ shore period cepherd with a period of about five days at the blue loop tip. The upper blue ioop is traversed very rapidly after the end of core helium burning. Notice that the mass is very reusonable and that the $7=0.02$ vilue of the composition is normal. For lower luminosities and periods at the blue loop tips, the luminosity necds to be as low as lop $\left(1 . / L_{a}\right)=3.0$ which orcurs with a $5 \mathrm{M}_{\mathrm{H}}$ model with a $\%$ of $0.0 ! 7$. The very lurge number of the double-mode Cepledes (maybe one in four hetween periods of 2 and 5 days and almost onc in 1 wo belween 2 and 3 davs $)$ points $: 0$ cvolution tracks with a slight mass dependent $T$, older stars hivill a lower $Z$, to give the long-lived blue loop lips (double-mode regime?) al the observed effective cemperature for hll thence double-mode stars 'lo get the even hoterer cepheid sill cas at an effective temperature of over $6300 \mathrm{~K}$, one meede ha even lower Z value, or perhape more rensollably nu ans ignement of this 1,95 day period Ceplesid as a first crossing Nent. These lideks come from the libraly of tracks of Hecker and Mathews, and the assumplion here is for no core overshootillg. It there 


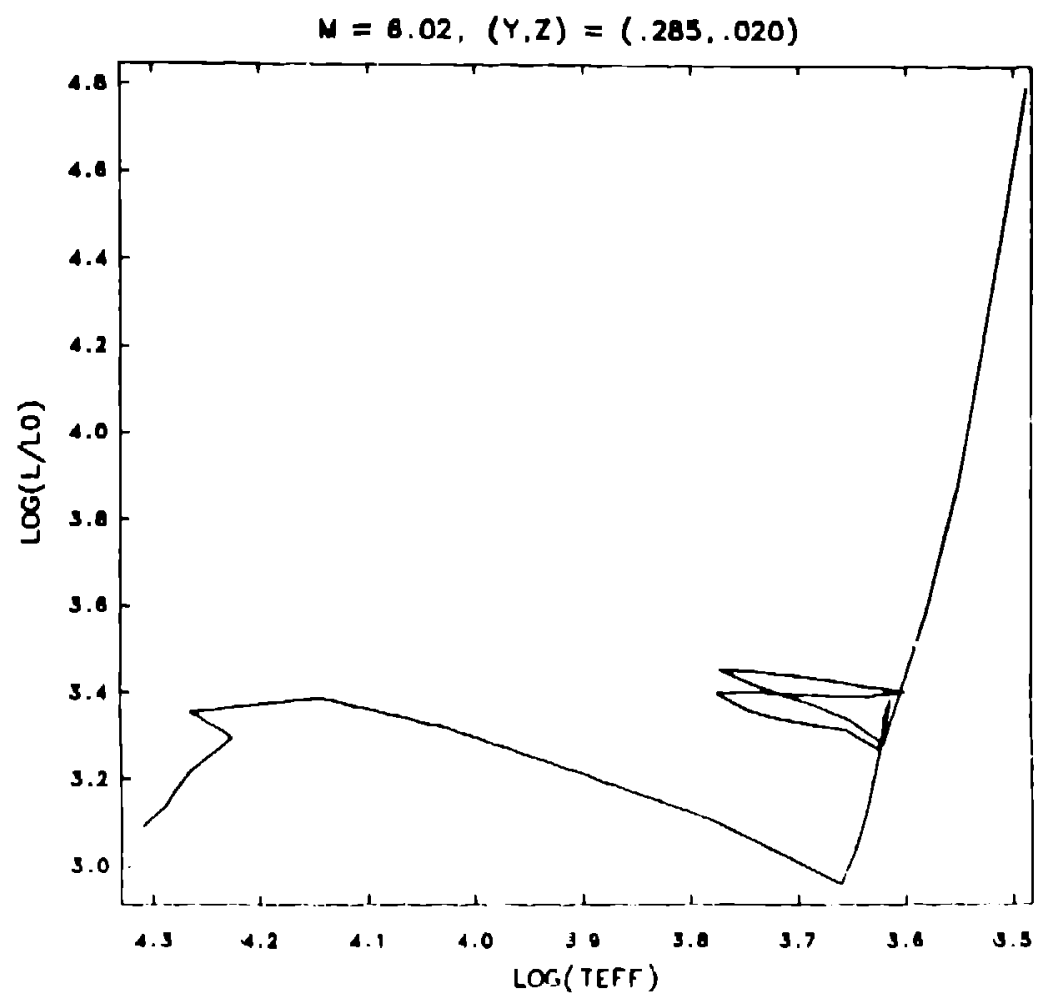

Figure 3. The evolution track for a $6 \mathrm{M}$ model with the $Y$ and $Z$ compositions, respectively, $0.285^{\circ}$ and 0.020 . The blue loops have their tips just at the effective temperature observed for the double-mode Cepheids.

Ss a little overshooling at $5 \mathrm{M}$, then the $Z$ value necds to be ever: lower chan 0.017 , bul not excessively so.

There is an unsolved problem leere, though. Pacaynski (1976), Robertson (19'1), and Robertson and Faulker (1972) have considered the possible" overshooting of the core convection on the blue loops in the core helium burning otage. This overshooting extends the blue loops and may upset our argument atout the $Z$ value for the composition. One nice feature cf their work is that with this overshooling during the blut loops, an even larger fiction of ims is spent at the blue tips. With all the proper effecti included, we may find that double-mode cepheids naturally occur at the hise lei tips for a conventional composition. The final resuits of Rotertson (1973) need further discussion, enpecinlly since the large oparty increases over the Cox and stewirt (196.5) values no longer reem just ified.

Turning now to anotlicr elass of variable stars, Saio and Whereler (1983) have considered the cosetraint of pulsation theory on the mass of three well known $R$ lirk stars. These hydrogen-delicient atars presumably bave lont their hydrogen envelope by a high mass lons rate cither from a rother massive star, bhy lo - is Ma, or from an older, lese massive, at ar evolving through the neymptotic giant branch region. 
Figure 4 shows the blue edge for the fundamental radial pulsation mode on the Hertzsp ng-Russell diagram for 1,2 , and $3 M_{0}$. The cause of the sharp turn of the blue edge towards higher effective temperatures at a critical luminosity is that the envelope density there becomes very low. At this mass-dependent critical luminosity the radiatively damping region of the star loses its effectiveness, while the cyclical ionization of helium even nearer to the surface still causes pulsation driving.

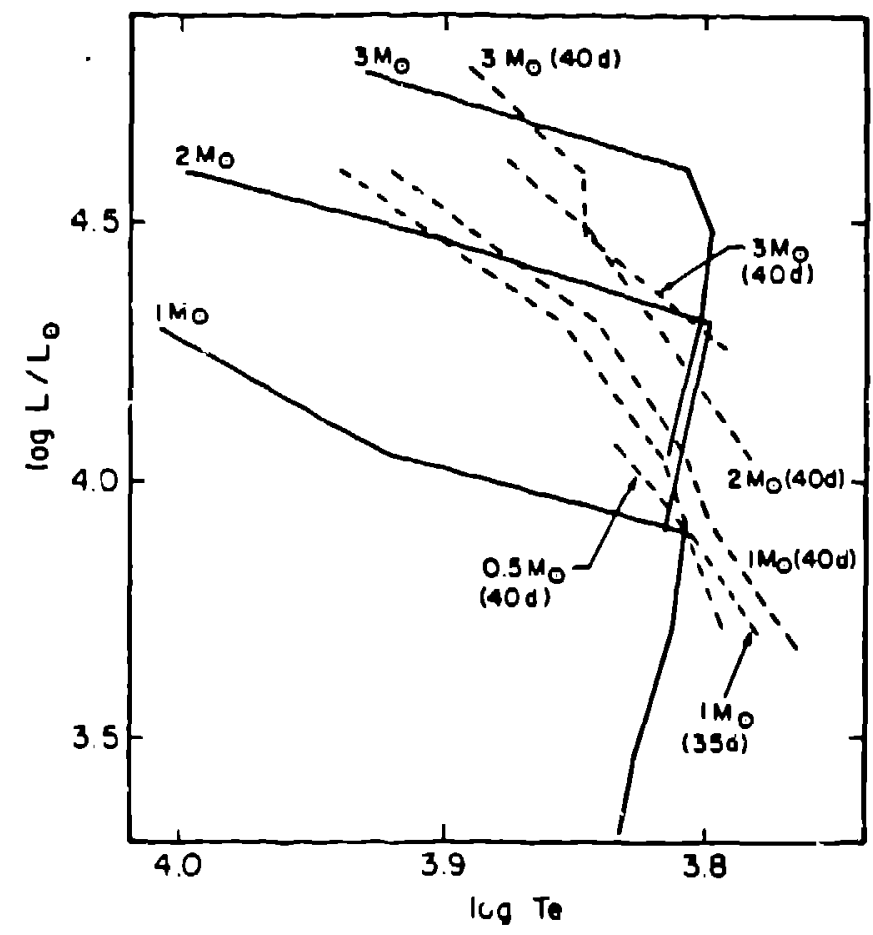

Figure 4. The Hertzsprung-Russell diagram in the region of the $\mathrm{K} \mathrm{CrB}$ variables. Blue edges are marked as solid lines for 1,2 , and 3 $M_{\text {G }}$. Also given are lines of constant period for masses and périods an marked.

The figure also gives dashed lines for the constant periol of 40 days and one case for 35 days. For the 3 M case, we can see the sudden increase in luminosity near $108\left(1 . / \mathrm{L}_{\mathrm{g}}\right)$ of 4.5 due to mode bumping. This bumping occurs when star:i become very centrally concentrated at high luminosity and periods decrease compared to those for less concentrated models. This decrease of a so-called longer period ntrange mode period makes it close to that for the fundamental which is less decreased in its period. Therefore, the potential collision of the two periods is oolved by the star when the fundamentel period is bumped down in period. This means that in retain the 40-day period, the radius and luminosity nee' to be increased suddenly.

The observes periods and aurface effective temperatures for $k$ bill and RY Sgr are 44 and 39 days and $7000 \mathrm{~K}$ and $7100 \mathrm{~K}$. This first clective 
tenperature is uncertain by only $250 \mathrm{~K}$, whereas the second has its uncertainty as $600 \mathrm{~K}$. XX Cam has similar properties, but it does not pulsate, probably because its luminosity is just a bit lower than for the other two stari and it never enters the pulsation instability strip.

Mass and luminosity limits can be obtained for the two variable stars by noting where the line of the observed period intersects the nearly horizontal blue edge at possible effective tenperatures of log $T$, between 3.829 and 3.860 for $R \quad C r B$ and 3.813 and 3.886 for $R Y$ Sgre Above about $3 \mathrm{H}$ the variable stars would not be predicted to pulsate because the line of constant period of 40 days is always in the stable, less luminous, region for all possible effective temperatures. Since RY Sgr can be a little bluer than R CrB, its mass and luminosity may pos ibly be a bit higher. For lower masses, the lines of constant period always are in the unstable region. In these cases, the luminosity limits always come from the observed effective temperature limits with, of course, a larger range for $R Y S_{g r}$.

Figure 5 presents the relation between the mass and luminosity limits. At low mass, if it all is in a helium core, there is an upper limit co the luminosity that can be produced according to Paczynski (1971), and that is the dash-dot line. The Eddington linit for the luminosity at any given mass is the upper solid line. Recently Coterell and Lambert (1982) have given for $R$ CrB a value for the gravity that can be combined with the measured effective temperature to constrain the massluminosity relation, and that is the heavy dotted line. We ce that the twu F CrB variables that pulsate have masias between about 0.8 and $3.1 M_{\theta}$ and luminosities between $\log \left(L / L_{\theta}\right)$ of 4.1 to 4.8. Further work on nonlinear pulsation calculations is now being done by Saio

We now turn lo some constraints from pulsation thoory for $\delta$ Scuti variables. Here we consider the slow settling of lelium below the botzom of the hydrogentheliun ionizatioll convertion zones. A $2 M_{\text {g }}$ star evolves slowly enough that this drainillg of helium can onerate even in the presence of mixing processes auch as those from rotation and curbulent diffusioll. During this stage the conviction zones remain homogeneous due to the strong motjons of convection, but the helidn content stendily decreases. When the helium mass fraction decreases lo aboul 0.08 , then the deeper helium convection zune dinappears. Al Lins low helium rontent the opacity is now low enough so that all the inteinally generated Iuminosity can be carried hy radiation alone. However, the ouler hydrogen convection zone is, if anylling, slightly enlarged becauge the high opncily of the hydrogen-lielium mixture is even now increased with less dilution by hrliun which does not contribute so mucli to the opacity at $10,000 \mathrm{k}$.

New evolution tracksi have bern computed by Andreasen, Hejlesten, null Peteraen (1983) allowing the heliun ta sette to depths of about $300,000 \mathrm{~K}$ and $1,000,000 \mathrm{~K}$. As one might expert, the tracks are not greatly changel on the Hertagprung-Rungell diagram. However, thene. 


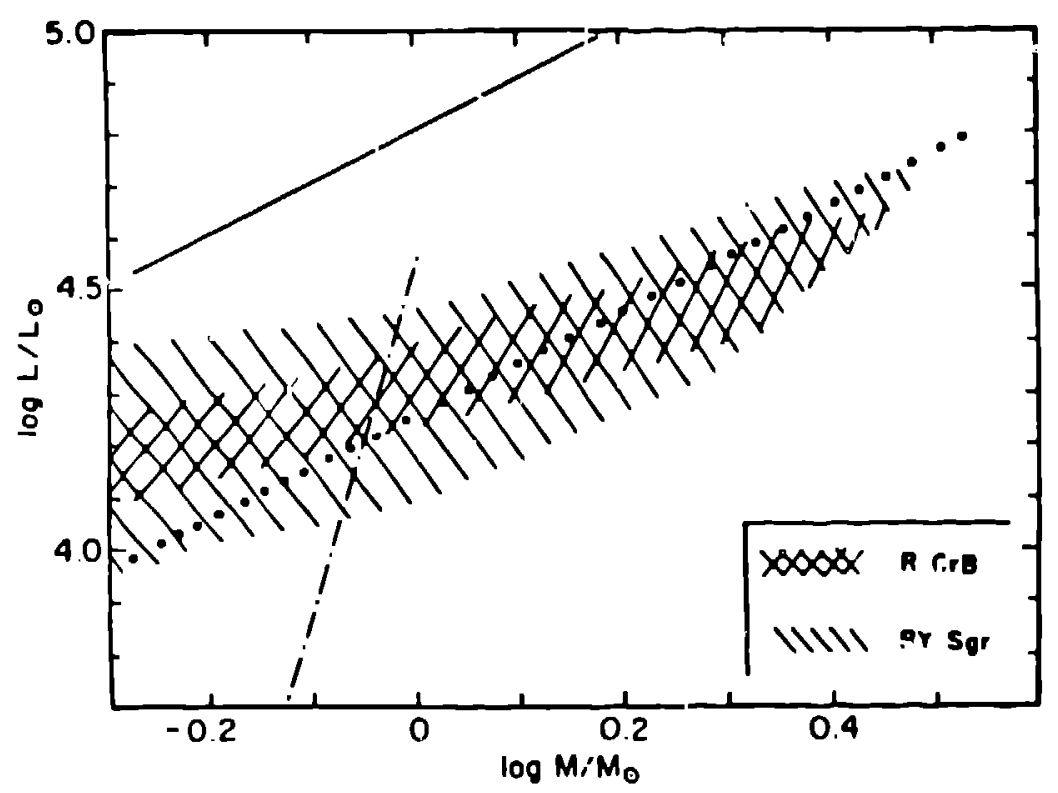

Figure 5. The allowed regions of mass and luminosities for $R$ CrB and RY Sgr from pulsation theory are given as hatched areas. The maximum luminosity as a function of mass is also drawn for low masses. The colid line is the Eddington limit as a linear function of mass. The dotted line is the mass-luminosity relation derived from the observation of the effective temperature and gravity of P. $\mathrm{CrB}$.

authors have found that as the stars expand in their normal evolution and their pulsation periods increase, the period ratio of the first radial overtolle to the fundamental increases to much larger values chan ever considered before. Their period ratio reaches 0.79 compared to just over 0.76 for 1.7 to $2.0 \mathrm{M}$ when the helium is denfeted down to about $300,000 \mathrm{~K}$. Deeper helium depletion returns the period ratio back lo or even slightly lower values.

This behavior of the overtone to fundamental period ratio is jus 2 as expected from the studies by Cox, King, and Hodson (1979) for decreasing the period ratios for double-mode Ceplieids. In that case, surface enriclment was needed to reduce rie ratio by about 0.01 lo 0.05 for masses, respectively of 6 and $4 M_{\theta}$. As discussed above, the physical cffect to change the period ratio"is a change in the apparent concentration of the star as seen differently by the two modes. For the double-mode. Cepheids the enrichment seems best for depths of $250,000 \mathrm{~K}$, not tno much deeper than we will see is appropriate for vi Cnc our $\delta$ Scuti varialile.

With McNamara from New Mexico Stale University, l began wondering if the large-period ratio observed for the 0.178 day $\delta$ Scuti variable VZ Cnc might indicate this helium setting. This star has been robserved 
recently by McNamara to compare with data obtained by Fitch (1955). Botb observers get the ratio to be 0.8006 , but the modern data give clearly a change in the amplitude ratio of the two modes with the overtone now $0.326 / 0.276$ larger relative to the then existing fundanental amplitude. The accuracy of the periods thenselves is not great enough to detect the few parts in a million perioo increase expected in the 30-year time interval, and therefore, a check that the expected redward evolution is really occurring is not yet possible. Anyway, it appears that the overtone is getting appreciably stronger rather than weaker as the star evolves to the red and to a more pure fundamental mode domain.

Figure 6 gives the period ratio being discussed for five calculated depths of the helium depletion in a model for a $2 M_{\theta}$ star with the observed surface effective temperature and a luminosity such that the observed period is matched to the radial fundamental. To reproduce the observed period ratio for VZ Cnc, a depletion profile is needed that has a surface helium mass fraction, $Y$, of either 0.00 or 0.08 down to almost 200,000 K. Deeper the $Y$ increases to 0.08 until a depth is reached where the Lemperature is almos $300,000 \mathrm{~K}$. Then we set $\mathrm{Y}=0.18$. Finally $Y=0.28$, the normal value, is used for depths deeper than about $400,000 \mathrm{~K}$.

The apparently correct interpretation for $V Z C n c$ is that its helium has settled to below $200,000 \mathrm{~K}$. This makes the blue edge of the instability strip for such a star just at $7000 \mathrm{~K}$, and the red edge is also probably at about $7000 \mathrm{~K}$. Thus this star just barely has an instability strip, and it is driven by hydrogen $\gamma$ and $K$ effects alone. Note that as the helium settles, the fundamental mode blue edge evolves to the red due to the depletion of helium. When a star is at or near this

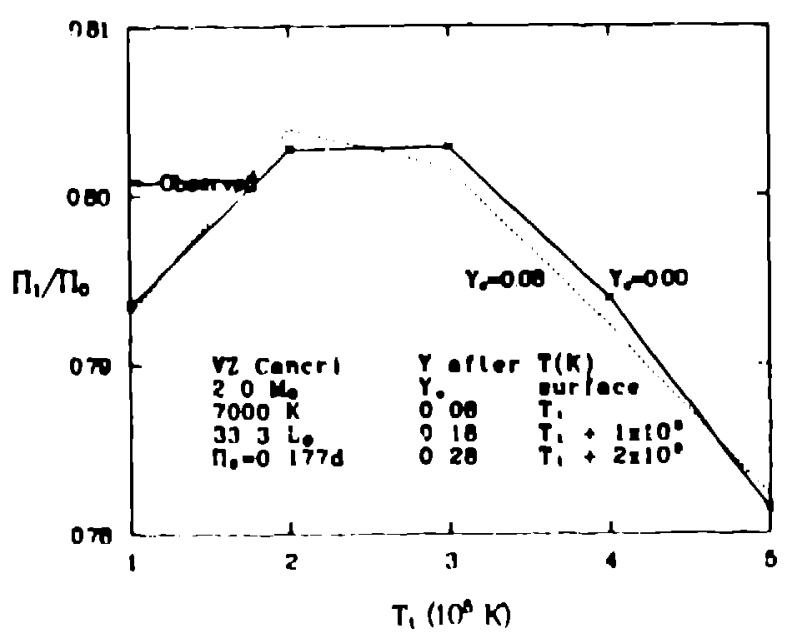

Figure 6. The period ratio vergus transition temperature is plotted for five depths of ettling of helium in a $2 \mathrm{M}$ model at $7006 \mathrm{~K}$ with the priod of 0.177 day. Two surface helium content mass fractions have becen considered. The observed period ratio is also indicated. 
blue edge there is a strong tendency to move over to an overtone pulsation as we see for double-mode Cepheids and double-mode RR Lyrae variables. It appears that this star has a redward evolution of its fundanental mode blue edge that is faster than the evolution of the star itself to the red, because the overtone mode is getting stronger. Helium depletion to depths such as we have found seem reasonable from the current results of diffusion theory.

One of the current interests in the constraints of pulsation theory on evolution theory is in the solar 5 minute oscillations. The low degree modes with $\ell=0$ to 5 give sensitivity to the central temperature and dersity struccure, and that in turn bears on the persistent problem of the bigher than observed predictions for the neutrino flux from the sun. The reason for the sensitivity of the periods of low degree oscillations is that the spacing between nodes near the center of the sun for, say the loth overtone, is comparable to the horizontal distance between node lines when $\ell=1$ to 5 . Thus a change in the central conditions results in a change in the nodal structure which reflects itself in changes of periods.

Figure 7 gives the internal structure of a solar model calculated by us at Los Alamos. The logarithms of the temperature opacity, and density are plotted versus the external mass fraction. The central temperature for this model is about 15 million kelvin, and the central density is about $122 \mathrm{~g} / \mathrm{cm}^{3}$, both somewlut different from other recent models. These differences may be due to the equation of state of the solar material that we used. Some coulomb corrections for the pressure have been included, but maybe not in as elaborate way as Ulrich and his collaborators or Shibahashi and his collaborators have done. The very high opacity across most of the figure produces a convective shell down to a temperature of just over $2.5 \mathrm{million}$ kelvin.

Figure 8 shows the hydrogen composition mass fraction profile for four different models. The basic profile is due to Christensen-Dalsgaaru (1982) who has made an actual evolution calculation using slandard methods. The solid slepped line shows our Los Alamos model which attempts to track the Christensen-Dalsgaarumodel as well as possible. The differences are needed due to slight differences belween the equation of state and opacities. Note that we need a sinaller amount of helium everywhere and it is nnt clear whether the sun produces that $10 \%$ gmaller amount during its lifetime. The figure also shows two partially mixed models, one with a homogeneous shell between $q=0.3$ and 0.5 and another with the central $15 \%$ of the mass homogeneous as shown. The pulsation periods for low degree, high overtone modes will later be calculated, and the effects of these two mixed layers on the periods will be given.

Observations of these modes in 1 h frequencies between 900 and $4500 \mu \mathrm{Hz}$ are given in Figurey. The plus signs, circles alld triangles give data for many of the modes, and the dots are the latert Shibahashi, Nocls, and Gabriel (preprint) thooretical results. The figure is really only 
Figure 7. The logarithms of the temperature, opacity, and density are plotted for our solar model versus sirface mass fraction. The units are: temperature $(K)$, opacity $\left(\mathrm{cm}^{2} / \mathrm{g}\right)$, density $\left(\mathrm{g} / \mathrm{cm}^{3}\right)$.

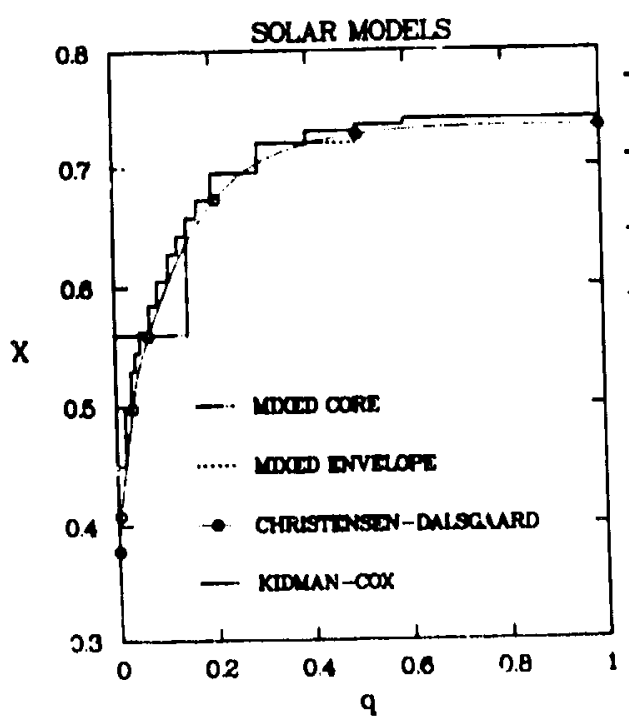

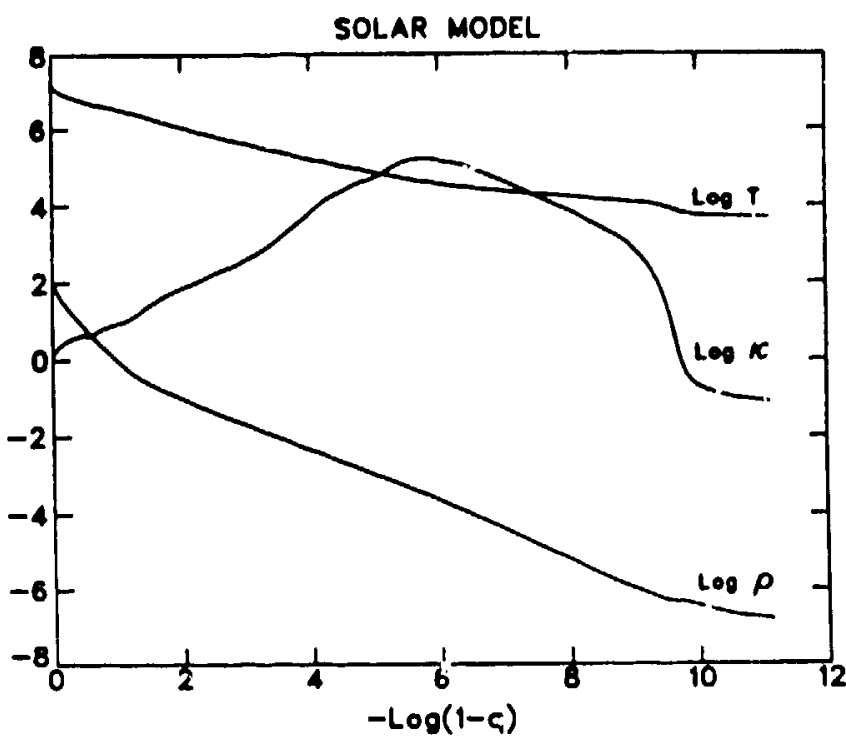

Figure 8 . Tile hydrogen mass fraction, $X$, is plotted versus internal mass fraction for four models including one with a mixed region in the envelope between $q=0.3$ and 0.5 and one homogenized in the central 0 . is of the mass.

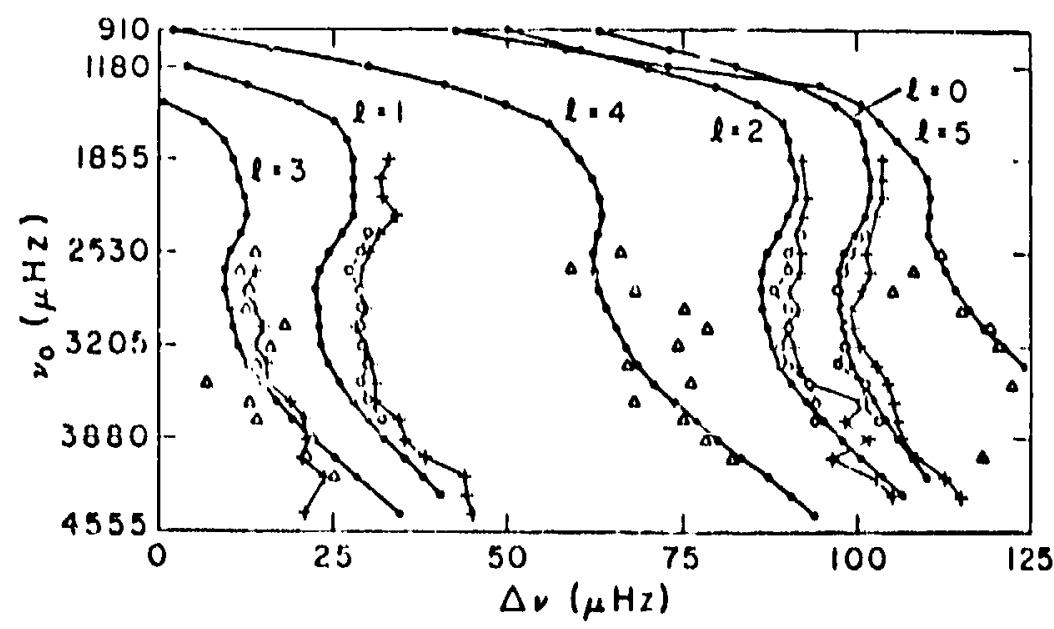

Figure 9. Frequencies of solar oscillations caleulated by shibahashi, Noels and cabrtel are ploted in 135 Mll sections and placed above one another. The observed nontadial modest are also given as presented by cinverie ol 11 . (1981) (opell circles), lires at al. (1980) (rroneck), and sherrir at al. (1982) (triankleg). 
one-dimensional, but the line of increasing frequency is cut into segments of $135 \mu \mathrm{H}$ and these lines are displayed one above the other. The match between theory and observation is good for these adiabatic periods. Our nonadiabatic periods are typically 0 to $7 \mu \mathrm{Hz}$ smaller in frequency. A thing to note about this figure is that the even $l$ values are close togecher and so are the odd ones if the $1=5$ curve had been plotted one raster line below. As shown by Vandakurou (1967) and recently by Tassoul (1980), the difference in frequency between two modes separated by +2 in $l$ and -1 in $n$ (overtone) is almost zero. The splitting of these modes is a good probe of the internal solar structure and its possible mixing either now or in the past.

The table of frequencies give our values for our slandard, mixed envelope, and mixed core modeis. We have been limited at the present time to only 330 mass she] $1 \mathrm{~s}$, but we believe that maybe 100 more zones are required to get accurate frequencies even for these longer than average periods, and lower than average order. Thus we get frequencies too large by 1.0 to $1.5 \%$. To approximately correct for this defect in nrder to assess the effects of the model structure on frequency splitting, we iave corrected all of our frequencies by the ratio of the measured to standard frequencies. This should reduce our splithing prediction errors to much less than $1 \%$.

The second table, with the separation of successive modes at a $r$ ixed $l$ value, averaged ove. all the four overtones, and with the above mentioned $\ell$ splitting averaged over four pairs shows the effact of the two miyed models. Our models still beem to have the separations shightly too large, though there is apparently no sensitivity to the possible internal mixing as expected. This separation is determined by the neodel structure in the outer $10 \%$ of the mass. We do see that homegerizing the rnvelope shell seems to slightly increate the $\ell$ splicting. All even larger increase, an also discussed recently by Ul rich and khodes (1983), i" seen for the core mixed model. It secms that the " splittink is sensitive to the cone structure. Mixing increases the splitting, but our core spacial renolutioll is loo coarse to mitcll observed splittings. No mixing at all serems to be n more correct model for our sun.

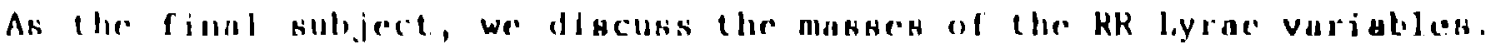
The discovery of the domble-mode KK lyrace variables In Ml! and MJ, das

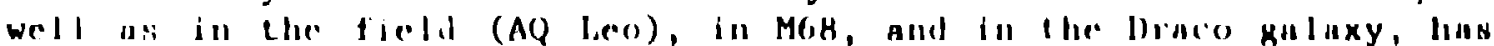

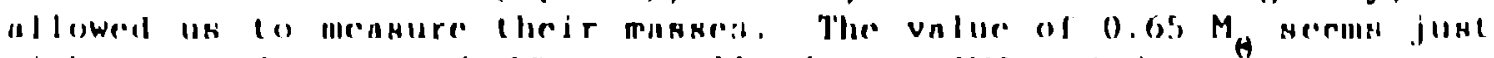

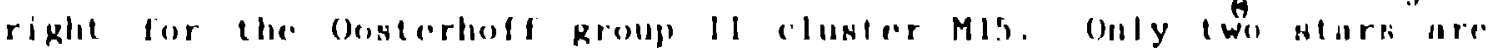

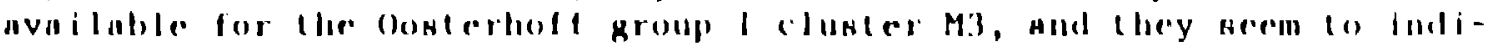

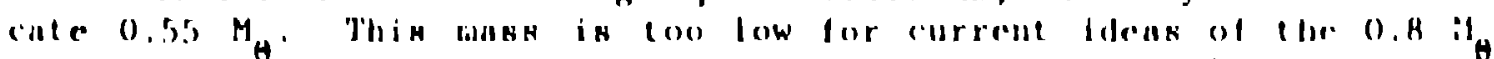

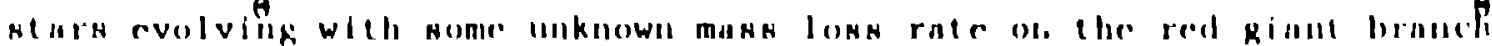
and lheir heliun-rich core mane when lliey experience the core tlash. It the low mane tor M3 in correct, there In netrong conet roint for red

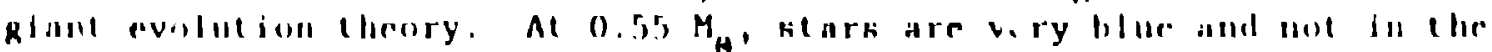

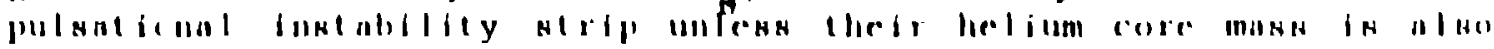

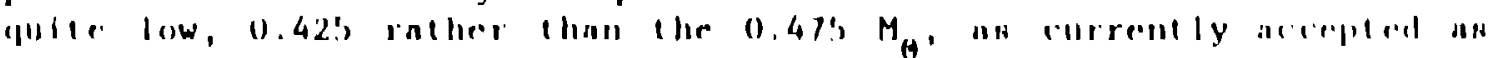
uproprertate. 


\begin{tabular}{|c|c|c|c|c|c|}
\hline DEGREE & ORDER & STANDARD & ENVILEPE & MIXED & EASURED \\
\hline 0 & $\begin{array}{l}10 \\
11 \\
12 \\
13 \\
13 \\
14\end{array}$ & $\begin{array}{l}15565 \\
17372 \\
1007 \\
2002 \\
2130\end{array}$ & $\begin{array}{l}1508 \\
1732 \\
1806 \\
2003 \\
2130\end{array}$ & $\begin{array}{l}1597 \\
1735 \\
1000 \\
20004 \\
2110\end{array}$ & $\begin{array}{l}1624 \\
1954 \\
2004\end{array}$ \\
\hline 1 & $\begin{array}{l}10 \\
10 \\
12 \\
13 \\
14 \\
14\end{array}$ & $\begin{array}{l}1846 \\
1762 \\
1010 \\
2060 \\
2103\end{array}$ & $\begin{array}{l}1046 \\
1702 \\
1910 \\
2060 \\
8103 \\
8103\end{array}$ & $\begin{array}{l}1045 \\
1783 \\
1920 \\
2067 \\
2104\end{array}$ & $\begin{array}{l}1753 \\
1768 \\
280202 \\
8187\end{array}$ \\
\hline 2 & $\begin{array}{l}10 \\
11 \\
12 \\
13 \\
18\end{array}$ & $\begin{array}{l}1090 \\
1635 \\
1071 \\
2110 \\
2247\end{array}$ & $\begin{array}{l}1099 \\
1895 \\
1937 \\
2110 \\
2240\end{array}$ & $\begin{array}{l}1090 \\
1635 \\
1071 \\
21110 \\
2247\end{array}$ & $\begin{array}{l}1012 \\
1047 \\
2002\end{array}$ \\
\hline 3 & $\begin{array}{l}10 \\
11 \\
12 \\
13 \\
14\end{array}$ & $\begin{array}{l}1740 \\
1767 \\
2028 \\
22105 \\
2301\end{array}$ & $\begin{array}{l}1749 \\
11007 \\
2026 \\
2103 \\
2301\end{array}$ & $\begin{array}{l}1749 \\
17697 \\
2024 \\
20162 \\
2301\end{array}$ & \\
\hline 4 & $\begin{array}{l}10 \\
11 \\
12 \\
13 \\
14\end{array}$ & $\begin{array}{l}1708 \\
1034 \\
2074 \\
2013 \\
22362 \\
2302\end{array}$ & $\begin{array}{l}1794 \\
17934 \\
2007 \\
2218 \\
2362 \\
2302\end{array}$ & $\begin{array}{l}1704 \\
1934 \\
2074 \\
2272 \\
2012 \\
8361\end{array}$ & \\
\hline
\end{tabular}

Table 1. Frequencies of our solar model are given for the longer observed periods in the 5 minute region.

$$
\begin{aligned}
& \text { AVERAGF: CORRFCTED } \\
& \text { SLIPARATIONS ANI } \\
& \text { SPLITTINGS ( } \mu \mathrm{H} z) \\
& (\mathrm{N}=10 \cdots 14)
\end{aligned}
$$

\begin{tabular}{|c|c|c|c|c|}
\hline$\downarrow$ & $\begin{array}{r}\text { STANDARD } \\
\text { MODELIL }\end{array}$ & $\begin{array}{r}\text { MIXED } \\
\text { ENYbilolses }\end{array}$ & $\begin{array}{r}\text { MI XFin } \\
\text { COSk: }\end{array}$ & MASSUUKE:D \\
\hline 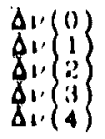 & $\begin{array}{l}134 \\
135 \\
135 \\
136 \\
137\end{array}$ & $\begin{array}{l}1: 14 \\
1: 16 \\
1: 36 \\
1: 30 \\
1: 34\end{array}$ & $\begin{array}{l}134 \\
136 \\
135 \\
130 \\
1117\end{array}$ & $\begin{array}{l}130 \\
136 \\
136 \\
1314\end{array}$ \\
\hline 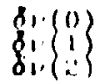 & $\begin{array}{l}3 \\
\vdots 1 \\
36 \\
36\end{array}$ & $\begin{array}{l}312 \\
112 \\
311\end{array}$ & $\begin{array}{l}3: 1 \\
3: 1 \\
37\end{array}$ & $\begin{array}{l}10 \\
\text { iil } \\
30\end{array}$ \\
\hline
\end{tabular}

lable 2. Separations and $\ell-s p l i t$ ling in whits of phr are piven for 8 values of 0 - 4 .

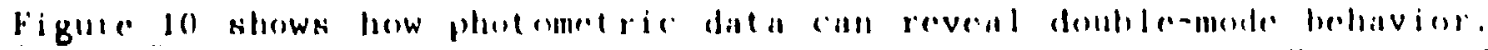

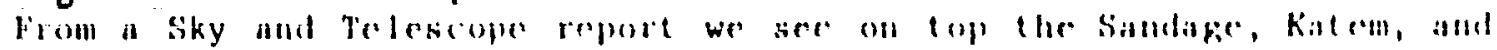
Sandage (1981) blue magultude data for lllt variable Vill. The period

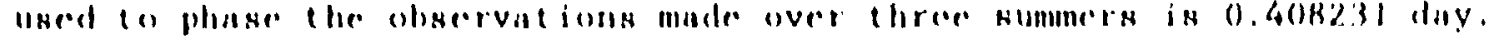

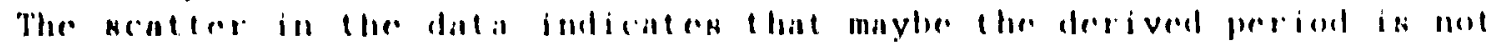

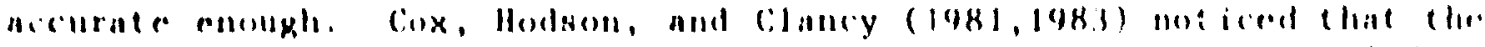

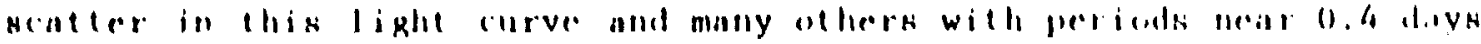

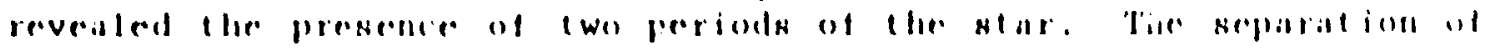

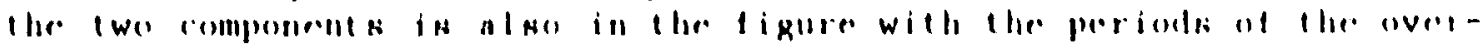

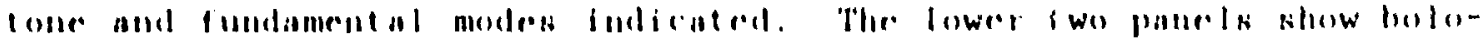

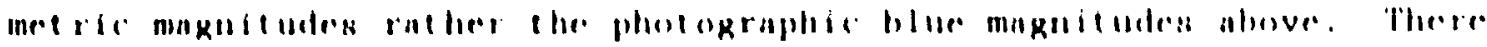

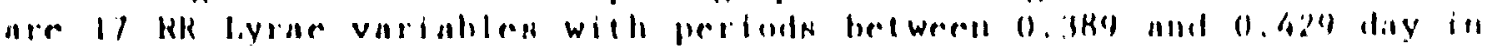




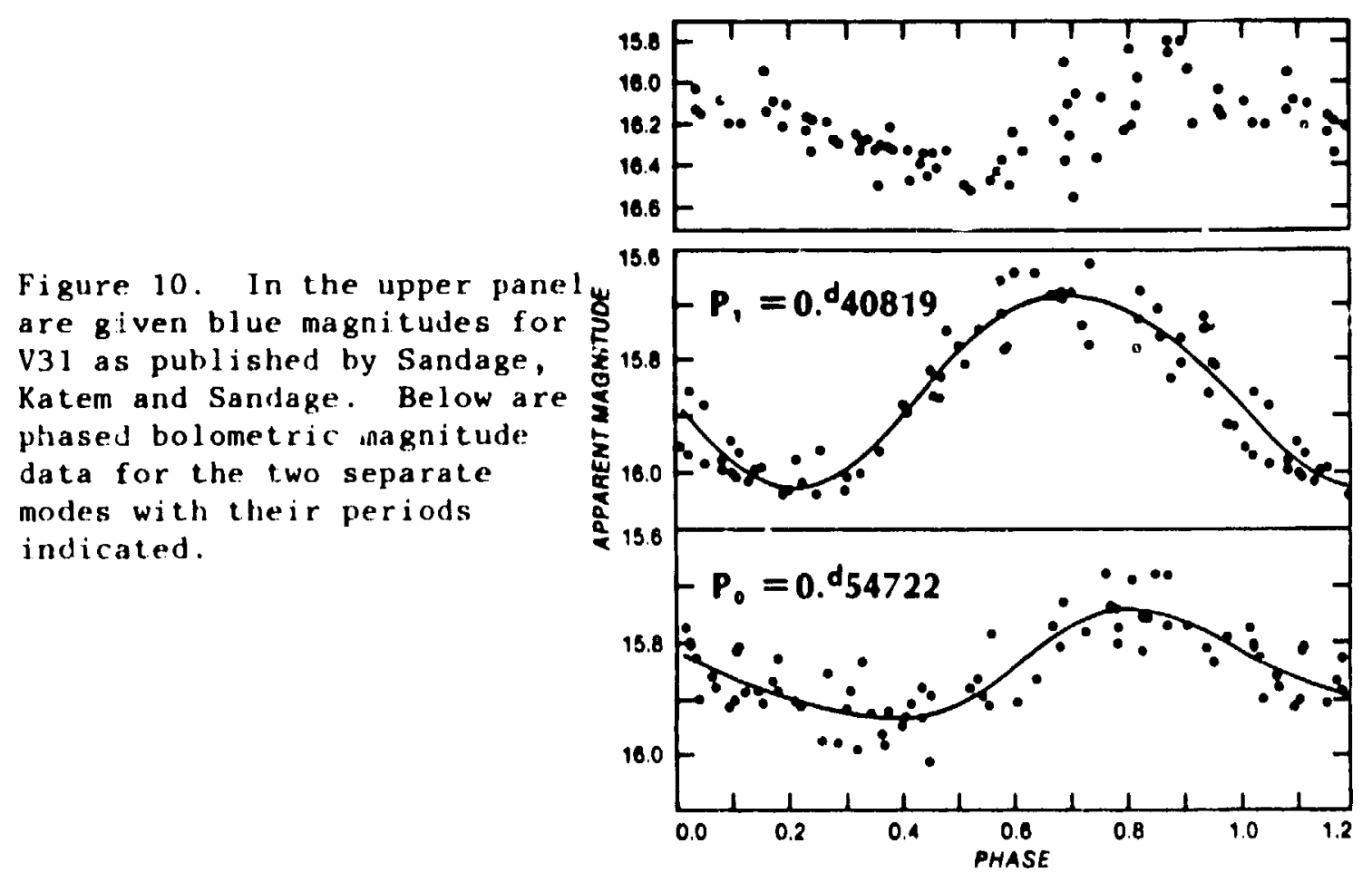

M15, and there are indications that all pulsate with two modes simultaneously. For the 12 cases where a good period ratio can be determined, the overtolle is always the stronger mode.

From ideas first presented by Jorgensen and Petersen $(1967)$, it is possible to use tlee pereol ratio to derive a mass for each star. This is pliysically possible because the two modes sample the outer structure differently due to their diflerent oscillation amplitude variations. the best way to portray this mass dependence is to show in figure 11 the period ratio versus period. This: diagram depende only si ight ly on the model luminosity and elfective temperature, and so it is usialde becanse it has bech chleulated for theme two parameters being rlose to lhose for renl RK lyrae variables. In ndelition to 12 Ml! valiables, we show also in the ligure the period ratio lor the ligela kk lyrae vari-

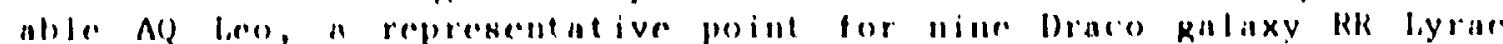

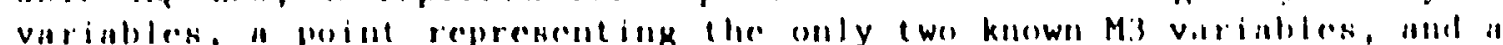

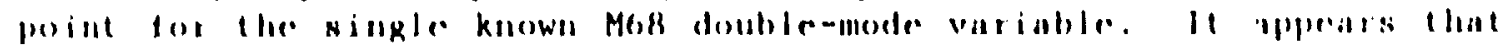
the Mls titars have a mane of 0.65 M as well as those nine lonly one

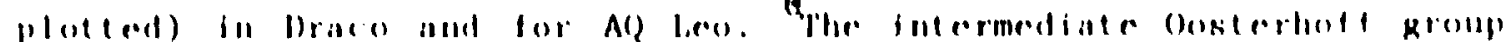

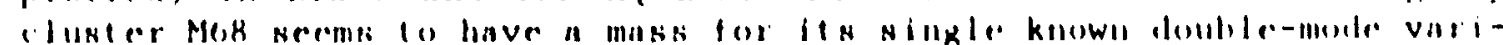

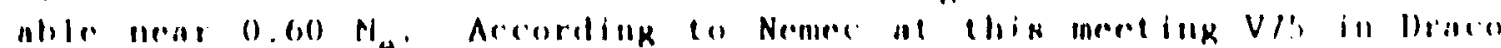

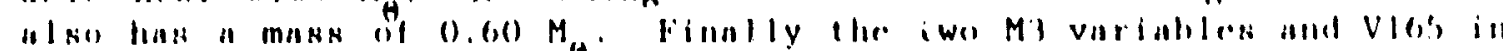

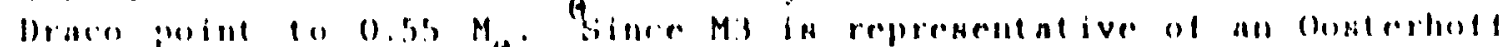

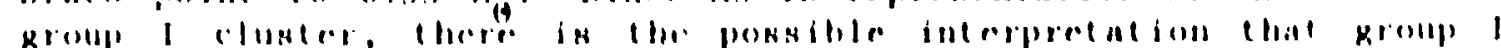

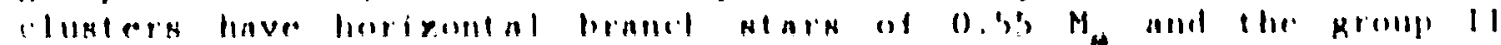

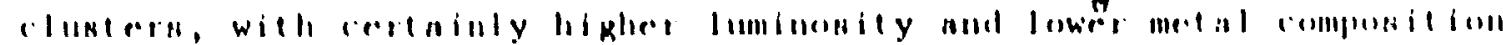

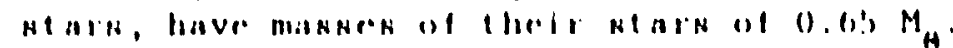


Figure 11. For all the listed double-mode RR Lyrae variables are plotted their period ratios versus their tundamental and overtone periods. The lines of constant mass are computed from linear nonadiabatic radial pulsation thenry.

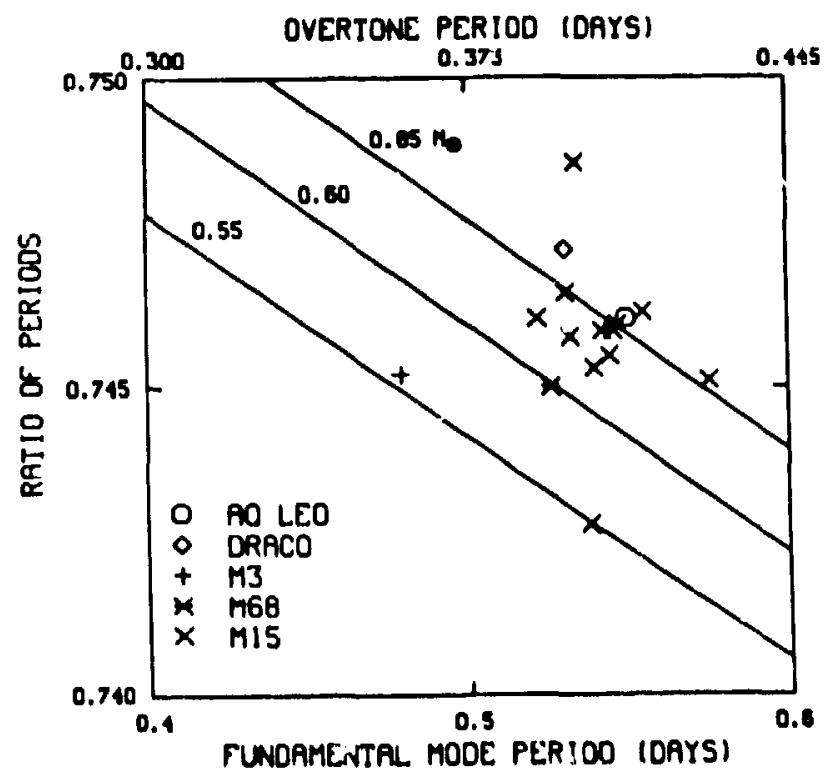

Witl. a mass of $0.65 M_{0}$, it is possible to make a theoretical Hertzsprung-Kassell diagram with the RR lyrae variable instubility strip blue and red edges shown. Figure 12 also gives the lines of constant fundamental mode period and lities of constant period ratio. A standard composition called king la has been used to derive the periocts and blue edges from linear pulsation theory. The red edge comes from the calculations of lleupree (19/7) which show how dependent the red edge is upon the helium content. On this diagram, we have plotted the mean luminosities of the MI! RR leyrae variables with f for lhe fumlamental mode variable: and o for the overtomes. The double-mode vari ables are plotted ns t signs. Here we luse onl the relative lumillosities and peri is as observed, The observed efeective temperntures are not used. A distance modulus of li.28 for MIS results in the luminosilies that are plotted. Posethle errors in the maghitudes and that from "change in mass by $0.0 \% M_{G}$ from the assumed 0.6! $M_{G}$ are indicated. The interesting thing ia that the the "ight plotted doublemerte variables

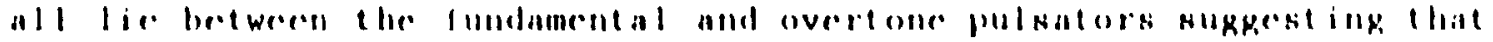
lhey are the renull ol nome kind of mods nwitebink.

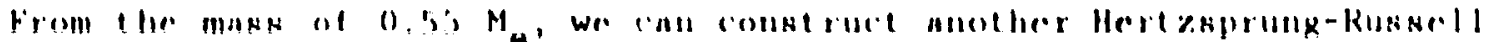

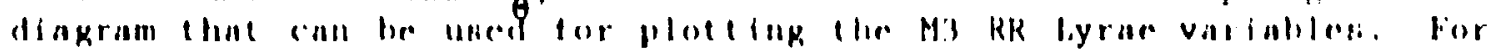

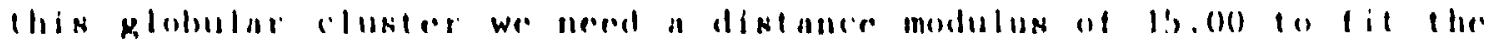
varialles into phe theoreteal tantability strif. This in Figure 13

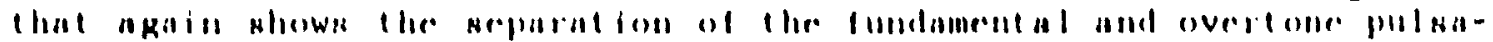

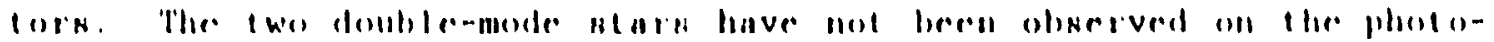

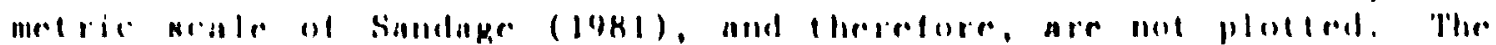

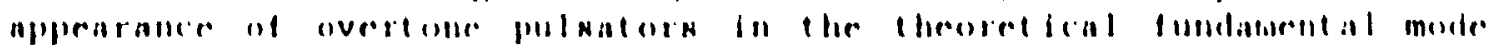

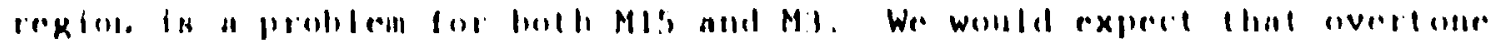

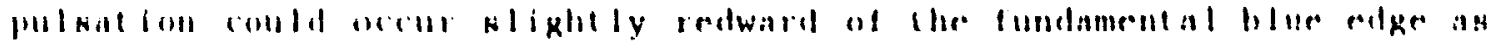

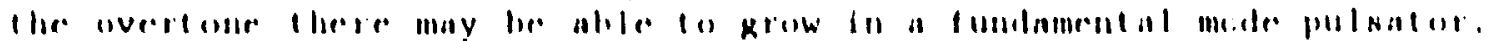


But the overtones and the double-mode pulsators seem to he much too red to match the best currently available blue edges and the fundamentalovertone transition line.

Figure 12. The RR Lyrae pulsation instability strip is plotted on the Hertzsprung-Russel] diagra..: for a mass of $0.65 M_{\theta}$ and the King Ia mixture composition. Mean luminosities over the pulsation cycle are plotted for the M15 RR Lyrae variables with the fundamental pulsators marked by $r$, the overtones by $D$, and the double-mode variables by + .
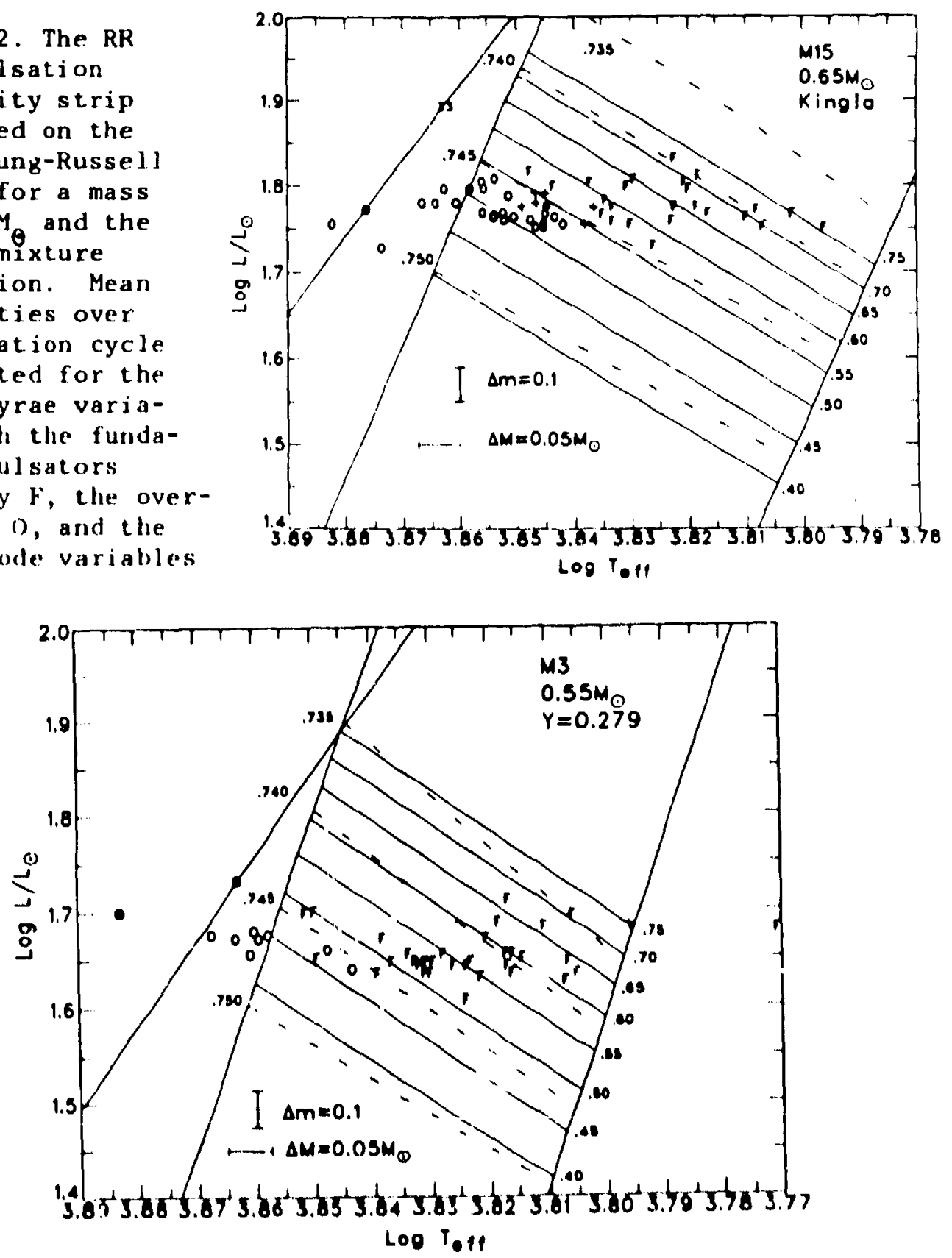

Higure 13. The RK lyrar pulsution instability serip is ploterel on the Hertenprung-Kunerll dingiam lor a mase of 0.5.5 M and l he mixture com-

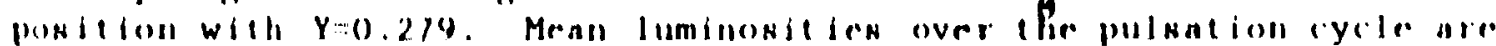

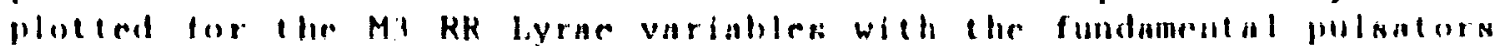

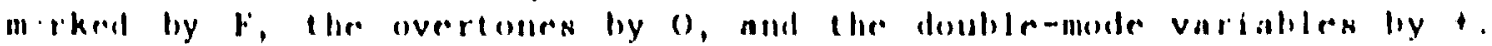


The constraint on evolution theory is that maybe the mass of the RR Lyrae variables differs with Oosterhoff groups, being lower in the higher metal content Oosterhoff group I clusters. Actually, with a core mass of about $0.475 \mathrm{M}_{\theta}$, as currently preferred, a mass of $0.55 \mathrm{M}_{\theta}$ would be born on the horizontal branch and evo've at much bluer positions than the instability strip. The only way to get RR Lyrae variables then is to reduce the helium-rich core to values as low as 0.425

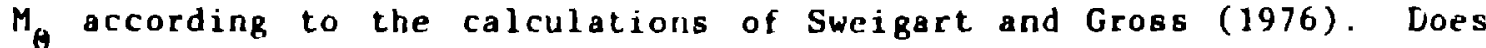
this mean that the higher $Z$ clusters have more mass loss and the core helium flash occurs at an earlier time in its evolution when less helium has been produced in the core?

We conclude from this discussion that there are some constraints on evolution theory from the various results of pulsation theory. The solar problem is interesting in that it reinforces our standard ideas of evolution, leaving the neutrino problem unsolved. The two other potentially strong constraints are from the double-mode Cepheids and the double-mode $\mathrm{RH}$ Lyrae variables. Future work for the first of these will be in theoretical studies of blue loop parameters. For the latter case, more accurate observations of RR Lyrae period ratios will evenLually define the horizontal branch star masses to produce very strong constraints for red giant and horizontal branch evolution calculations. 


\section{References}

Andreasen, G.K., Hejlesen, P.M., and Petersen, J.0. 1983, Astron. Ap. in press.

Balona, L.A. and Stobie R.S. 1979, M.N.R.A.S. 189, 659.

Barrel1, S.L. 1981 , M.N.R.A.S. 196, 357.

Becker, S.A. and Cox, A.N. 1982, Ap.J. 260, 707.

Bel1, R.A. and Parsons, S.B. 1972, Ap Letters, 12, 5.

Burki, G. 1983, Astron. Ap. 1983, in press.

Christensen-Dalsgaard, J. 1982, M.N.R.A.S. 199, 735.

Claverie, A., Isaak, G.R., McLeod, C.P., Van der Raay, H., and Roca Cortes 1981, Nature 293, 443.

Cottrel1, P.L. ara Lambert, D.L., 1982, Ap.J. 261, 595.

Cox, A.N. 1979, Ap.J. 229, 212.

Cox, A.N., Hodson, S.W., and Clancy, S.P. 1981, in IAU Colloquium 68, "Astrophysical Parameters for Globular Clusters," eds. A.G. Davis Philip and D.S. Hayes L. Davis Press, Inc, Schenectady, New York p 737 .

Cox, A.N., Hodzon, S.W., and Clancy, S.P. 1983, Ap.J. 266, 94.

Cox, A.N., King, D.S., and Hodson, S.W. 1979, Ap.J. 228, 870.

Cox, A.N. and Stewart, J.N. 1965, Ap.J. Suppl., 11, 22.

Peupree, R.G. 1977. Ap.J. 214, 502.

Fernie, J.D. 1979, Ap.J. 231, 841 .

Fitch, W.S. 1955, Ap.J. 121, 690.

Flower, P.J. 1977, Astron. Ap. 54, 21.

Grec, C., Fossat, E., and Pomerantz, M. 1980, Nalure 288, 541.

Hanson, R.B. 1977, in IAU Symposium 80, "The H-R Diagram" eds. A.G. Davis Philip and D.S. Hayes, Reidel, Dordrecht, p154.

Huang, R.Q. and Weigerl, A. 1983, Astron. Af. in press.

Jorgensen, H.F. and Petersen, J.0, 1967, Zs.Ap. 67, 377.

Luck, R.E. and LamherL, D. I. 1981, Ap.J. 245, 1018.

Maeder, A. 1981, Astron. An, 102, 401.

Mheder, A. and Mernilloid, J.C. 1981, Astron. Ap. 93, 136.

Matraka, B., Whagermann, C., alld Weigert, A. 1982, Astroll. Ap. 107, 283.

Paczynaki, B. 1970, fele Astr. 20, 195 .

Paczynaki, B. 1971, Acla Astr. $21,1$.

Fel, J. 1978, Aftron. Ap, Suppl. 31, 489.

Hobertson, J.W. 1971, Ap.J. 170,353.

Hobertson, J.W. 1973, Ap..J. 185, $8 ! 7$.

Roterenon, J.W. and Faulkner, 1).J. 1972, Ap.J. $171,104$.

Saio, H. ond Wheceler, J C. 1983, Ap.J. Lete. 272, 1.25.

Sandage, A. 1981, Ap.J. 248, 161.

Sandage, A., Katem, H., and Sandage, M. 1981, Ap.J. Suppl. 40, 41 .

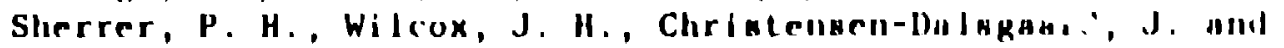
Gough, D. O. 19H2, Meprint.

Sweigart, A, V. and Grons, P.G. 1976, Ap..J. Suppl. $32,367$.

Tansoul, M. 1980, Ap... Suppl. 43, 469.

UI rich, K.K. and Khodes, E.J. 1983, Ap.J. 265, 5.51.

Vambikurov, Y.V. 1467, Ant.7h. 44,786. 